\title{
Radiation Induced Sarcoma of Head and Neck: A Rare Entity
}

\author{
Deepak Paudel ${ }^{1 *}$, Abhijit Gundale ${ }^{1}$, RajinikanthJanakiraman ${ }^{1}$ and Mandeep Bindra ${ }^{2}$ \\ ${ }^{1}$ Department of ENT \&HNS , BPKIHS, Dharan Nepal \\ ${ }^{2}$ Department of Head \& Neck Surgery, Christian Medical College, Vellore India
}

\begin{abstract}
Sarcoma are rare in head and neck region and radiation induced sarcoma (RIS) are even rare and poses challenges for diagnosis and proper management. We report a case of 38 year old male who was treated for carcinoma of right lateral border of tongue with surgery and radiotherapy, and presented with rapidly progressing mass in right floor of mouth after 4 years. Biopsy and imaging confirmed the diagnosis of radiation induced sarcoma and was managed with wide local excision of mass with hemimandibulectomy with reconstruction by pectoralis major mayocutaneous flap. Patient completed the follow up of one and half year and doing well.

RIS of head and neck is rare entity and demands multidisciplinary involvement of head and neck, neuro, reconstructive surgeons, radiation oncologists and medical oncologists for optimal management. Though it has poor prognosis but properly managed patients will have the good quality of life.
\end{abstract}

Keywords: Radiation Induced Sarcoma (RIS), Head and Neck Tumor, Post Irradiation

\section{Introduction}

Head and neck sarcomas are relatively rare clinical entities, $10 \%$ of sarcomas in adults_originates in the head and neck region accounting for $1 \%$ of all head and neck malignancies, radiation induced head and neck sarcomas are even more rare and the incidence range from 0.16 to $0.3 \%$. $(1,2)$ Radiation is the integral part of head and neck cancer management but has some deleterious effect including development of second malignancy at the site of exposure. A squamous cell cancer is the commonest histologic subtype of radiation-induced malignancy in the head and neck region followed by radiation induced sarcoma(RIS) which consists of $12 \%$ of radiation induced malignancy and estimated life time risk is of 0.03 to $0.3 \%$ in post-irradiated patients. Radiation induced sarcoma is on constant surge due to the improved survival after radiotherapy, the development of RIS depends on factors like dose of radiation, age of treatment, use of chemotherapy, and other genetic tendencies of the patients.(3)

Though there is no side predilection for RIS in head and neck region more than $80 \%$ of them developed in survivor of Nasopharyngeal carcinoma. (2) We present the rare case of RIS of floor of the mouth who was treated for squamous cell carcinoma of right lateral border of tongue by primary surgery followed by adjuvant Radiotherapy.

\section{Case Report}

A 38 year old gentle man was evaluated for rapidly developing swelling in right side of floor of mouth in April
2016 during his regular follow up visit. He was a diagnosed case of squamous cell carcinoma of right lateral border of tongue with clinical stagingT2N0M0 and managed with wide local excision with right selective neck dissection including level I to IV with radial artery fore arm free flap. Patient received adjuvant radiation as final histopathology report showed lympho- vascular invasion, he received 70 Gy of radiation in 35 fractions over the period 7 weeks. The treatment was completed in March 2012 since then he was on regular follow up and he was alright in his last follow up six months back. Though he was ex cigarette smoker and smokeless tobacco chewer he did not resume the habit after the treatment.

On examination he had a firm to hard swelling in right side of floor of mouth measuring $4 \times 4 \mathrm{cms}$ and involving the right lateral part of tongue (flap region) and was $2 \mathrm{~cm}$ from the midline and reaching the tip of the tongue. Posteriorly it reached till the junction of anterior $2 / 3 \mathrm{rd}$ and post $1 / 3$ of tongue. There were no clinically palpable nodes. Patient was advised for PET-CT scan which showed mass in right side of floor of the mouth abutting the mandible (Image 1) and was continuous with level IB node and also involved the right masticator space and there was no distant metastasis. Pre-operative biopsy showed features of radiation induced sarcoma.

As there was no distant metastasis and patient was fit for surgery, he was treated with wide local excision with right hemimandibulectomy and reconstructed with pectoralis 
major mayo cutaneous flap in May 2016. Post-operative histopathology showed high grade radiation induced sarcoma suggestive of undifferentiated pleomorphic sarcoma. (Image 2) He received adjuvant radiotherapy and systemic therapy, and doing well after one and half year of treatment (Image 3)

\section{Discussion}

Radiotherapy is one of the main modality of treatment of head and neck cancer, used in definitive or adjuvant settings. With improved survival of head and neck cancer,

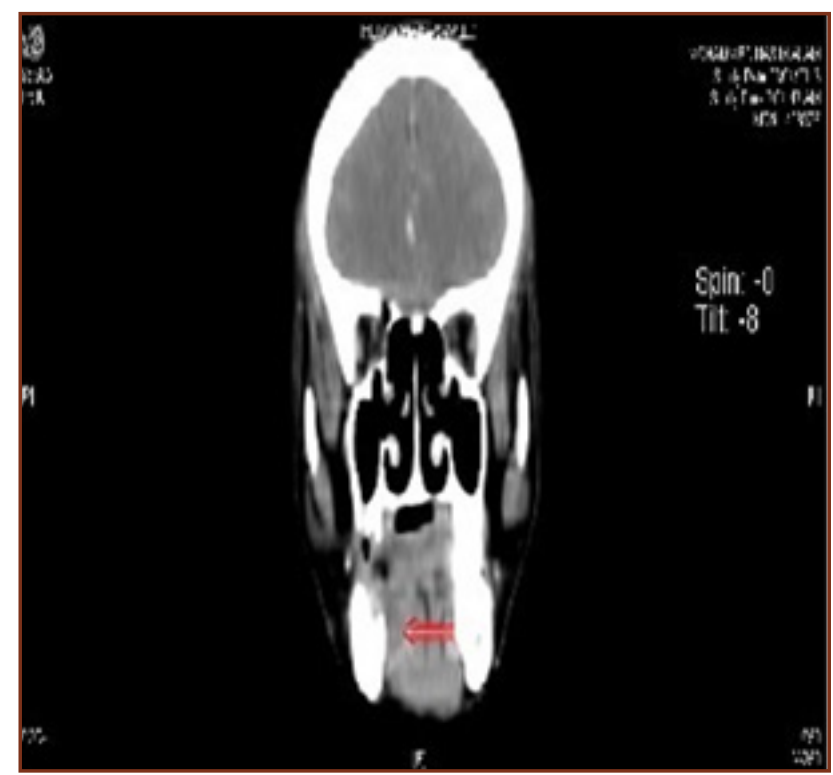

Fig. 1: Pre-op CT Scan showing mandible abutment. increased attentions should focuses to the treatment related morbidity as well. Radiation-induced second malignancies particularly radiation-induced sarcomas are the most devastating of the late complications of radiotherapy. (3) Pathophysiology of RIS is poorly understood and proposed mechanism is Radiation induced DNA damage leading to $p 53$ point mutations.and genetic aberrations in the $\mathrm{Rb}$ gene. Beside mutagenic effect, radiation changes the microenvironment which promotes genomic injury in stem cells and enhances the expression of a neoplastic phenotype..(3,4) By stander effect, transmission of

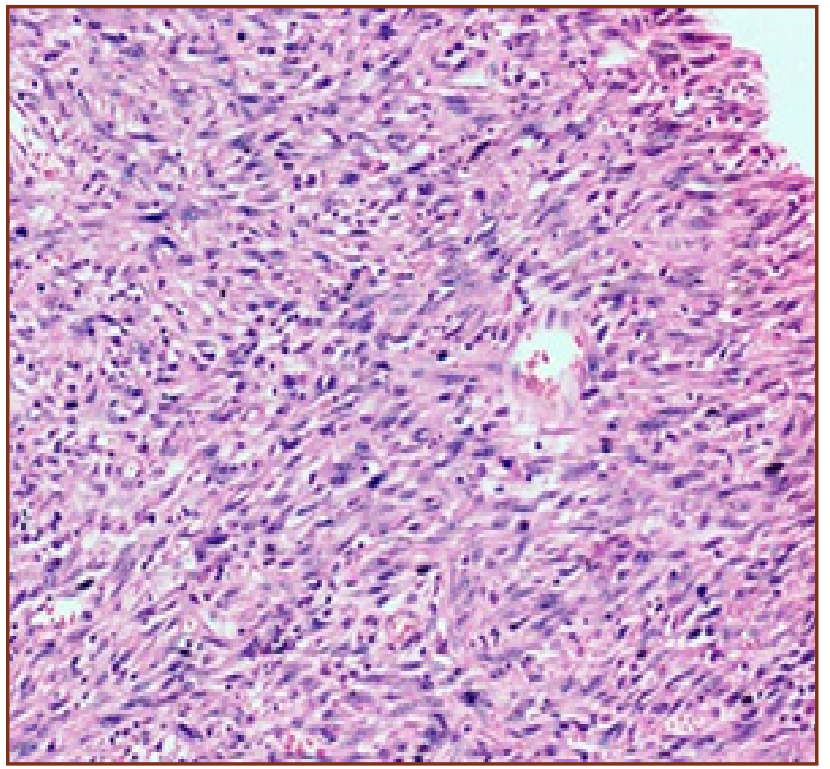

Fig. 2: H\& E section under 40x.

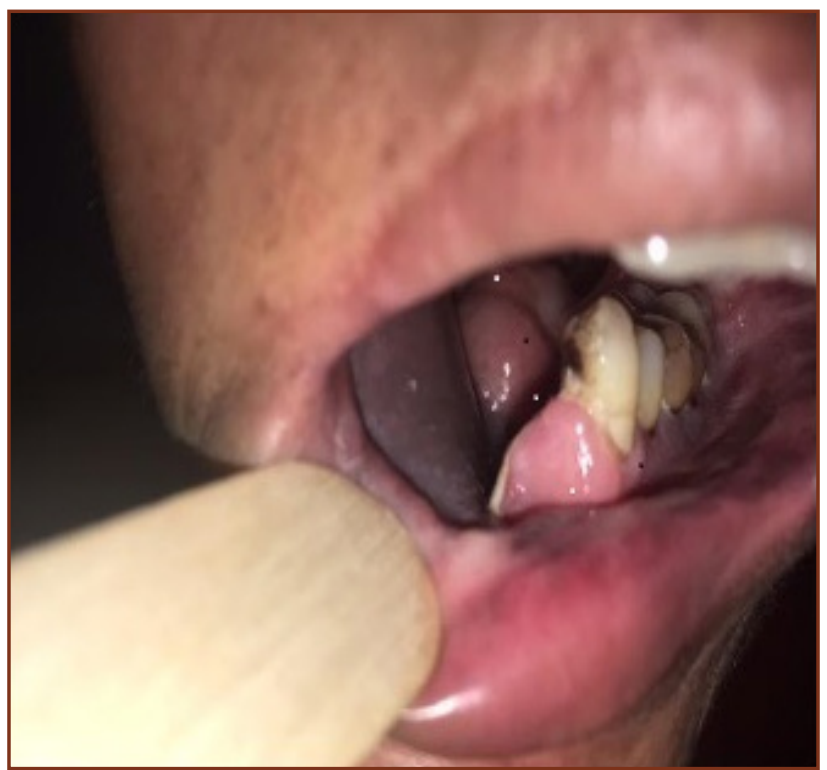

Fig. 3: shows the post of picture with health flap in floor of mouth. 
signal between irradiated (in-field) cells and neighboring unirradiated cells can promote the development of persistent reactive oxygen species in unirradiated cells and hence, tumorigenesis, is also proposed as a possible mechanism of development of RIS. $(5,6)$

Sarcomas are second most common radiation induced malignancies after squamous cell carcinoma, RIS and sarcomatogenesis requires following conditions: (1) the sarcoma should arise within the irradiated field (in the area encompassed must be histologically distinct from the index lesion; and (3) there must be a latency of several years

between radiation exposure and subsequent diagnosis of the sarcoma.(7) This time interval included in the criteria to differentiate post-irradiation sarcomas from sporadic sarcomas that may have predated radiation therapy and Subsequent modifications made to reduce the time interval ranging from 6 months to 4 years.(8) For radiation induced head and neck sarcomas, arbitrary time frames of 3-4 years have been used as cutoffs based on a consensus that this much time is sufficient to carcinogenesis to occur.(9)

There are no specific histopathologic criteria to distinguish between radiation-induced sarcomas and sporadic sarcomas arising in the irradiated field except the features of radiation induced changes in the immediate vicinity of the tumor i.e. dense cellular fibrosis, atypical fibroblasts, alteration of the vascular architecture, and abundant fibrous stroma in the dermis.(10) Histologic subtypes of RIS are similar to that of de novo sarcoma which include osteosarcoma, chondrosarcoma, malignant fibrous histiocytoma, fibrosarcoma, angiosarcoma, synovial sarcoma, and malignant peripheral nerve sheath tumors and rhabdomyosarcoma in children.(11)

Optimal management of RIS includes multidisciplinary involvement of head and neck, neuro-, and reconstructive surgeons to maximize resectability and local control of the disease, radiation oncologists and medical oncologists to consider the role of re-irradiation and or adjuvant systemic therapy respectively. Adjuvant radiotherapy may have a role in treatment of RIS of the head and neck, but major limitation being the amount of prior radiotherapy in the same field. The benefit of chemotherapy for head and neck soft tissue sarcomas after optimal local therapy is uncertain and it is small if there any but control with adjuvant chemotherapy may be beneficial in case of un-resectable head and neck sarcomas.(12,13)Surgical management of RIS of region head and neck is challenging because its proximity to the vital structures and difficulties in operating in an irradiated field, but complete excision of the tumor along with appropriate reconstruction only offers long term survival and functional benefit.(11) The prognosis of
RIS is poor as compared to de novo sarcoma which could be due to (1) difficulties of diagnosis in irradiated field; (2) compromised resection margins (3) limited treatment option i.e difficulties of operating within an irradiated field and problem with reirradiation. $(14,15)$

This case was rare as the index tumor was of tongue contrary to the most other cases arises in the irradiated field of Nasopharyngeal carcinoma, and the causality between radiation and sarcomagenesis was established as 1; it arose within irradiated tissue2; it had distinct histology from index lesion 3it had prolonged Latency period and 4; was corroborated with core needle biopsy and imaging which clinched the diagnosis. The case was managed with upmost care as got the negative resection margin and patient also received adjuvant radiotherapy and systemic treatment, and he is doing well after one and half year of treatment.

\section{Conclusion}

Radiation induced sarcoma of head and neck region are rare, most of them arise in the irradiated field of nasopharyngeal carcinoma. It demands multidisciplinary involvement of head and neck, neuro, reconstructive surgeons, radiation oncologists and medical oncologists for optimal management. Though RIS has poor prognosis as compared de novo sarcoma but properly managed patients have the good quality of life.

\section{Acknowledgements}

We would like to acknowledge Mr Kantha Rao, head and technician, who provided the images of the patient.

\section{Reference}

1. Rosko AJ, Birkeland AC, Chinn SB, Shuman AG, Prince ME, Patel RM, et al. Survival and Margin Status in Head and Neck Radiation-Induced Sarcomas and De Novo Sarcomas. Otolaryngol--Head Neck Surg Off J Am Acad OtolaryngolHead Neck Surg. 2017 Aug;157(2):252-9.

2. Yeang MS, Tay K, Ong WS, Thiagarajan A, Tan DS-W, $\mathrm{Ha} \mathrm{TC}$, et al. Outcomes and prognostic factors of postirradiation and de novo sarcomas of the head and neck: a histologically matched case-control study. Ann Surg Oncol. 2013 Sep;20(9):3066-75.

3. Thiagarajan A, Iyer NG. Radiation-induced sarcomas of the head and neck. World J Clin Oncol. 2014 Dec 10;5(5):97381.

4. Barcellos-Hoff MH, Nguyen DH. Radiation carcinogenesis in context: how do irradiated tissues become tumors? Health Phys. 2009 Nov;97(5):446-57.

5. Mothersill C, Seymour C. Radiation-induced bystander effects, carcinogenesis and models. Oncogene. 2003 Oct 13;22(45):7028-33.

6. Goldberg Z, Lehnert BE. Radiation-induced effects in unirradiated cells: a review and implications in cancer. Int J Oncol. 2002 Aug;21(2):337-49. 
7. Cahan WG, Woodard HQ. Sarcoma arising in irradiated bone; report of 11 cases. Cancer. 1948 May;1(1):3-29.

8. Cha C, Antonescu CR, Quan ML, Maru S, Brennan MF. Long-term results with resection of radiation-induced soft tissue sarcomas. Ann Surg. 2004 Jun;239(6):903-909; discussion 909-910.

9. Huber GF, Matthews TW, Dort JC. Radiation-induced soft tissue sarcomas of the head and neck. J Otolaryngol. 2007 Apr;36(2):93-7.

10. Thariat J, Italiano A, Collin F, Iannessi A, Marcy P-Y, Lacout A, et al. Not all sarcomas developed in irradiated tissue are necessarily radiation-induced--spectrum of disease and treatment characteristics. Crit Rev Oncol Hematol. 2012 Sep;83(3):393-406.

11. Patel SG, See AC, Williamson PA, Archer DJ, Evans PH. Radiation induced sarcoma of the head and neck. Head Neck. 1999 Jul;21(4):346-54.
12. Blay J-Y, Le Cesne A. Adjuvant chemotherapy in localized soft tissue sarcomas: still not proven. The Oncologist. 2009 Oct;14(10):1013-20.

13. Shaheen M, Deheshi BM, Riad S, Werier J, Holt GE, Ferguson PC, et al. Prognosis of radiation-induced bone sarcoma is similar to primary osteosarcoma. Clin Orthop. 2006 Sep;450:76-81.

14. Gladdy RA, Qin L-X, Moraco N, Edgar MA, Antonescu $\mathrm{CR}$, Alektiar KM, et al. Do radiation-associated soft tissue sarcomas have the same prognosis as sporadic soft tissue sarcomas? J Clin Oncol Off J Am Soc Clin Oncol. 2010 Apr 20;28(12):2064-9.

15. Thijssens KMJ, van Ginkel RJ, Suurmeijer AJH, Pras E, van der Graaf WTA, Hollander M, et al. Radiation-induced sarcoma: a challenge for the surgeon. Ann Surg Oncol. 2005 Mar;12(3):237-45.

*Corresponding author:

Dr Deepak Paudel, Department of ENT \& HNS, BPKIHS, Dharan, Nepal

Phone: +97 79852056991

Email: d_pakh@yahoo.com

Financial or other Competing Interests: None. 